Opinion

\title{
Obesity and eating healthy: a positive approach
}

\author{
Abstract \\ General guidelines of following healthy eating by trying to include the MyPlate \\ guidelines and dealing with lifestyle change by turning to a RDN or DTR for nutrition \\ education.
}

Keywords: myplate, RDN, DTR, obesity, BMI, nutrition
Volume 2 Issue 5 - 2015

\section{Leah Firestone}

Clinical Dietetic Technician, Greensburg Care Center, USA

Correspondence: Leah Firestone, Clinical Dietetic Technician, Greensburg Care Center, 1628 BROAD ST Greensburg, PA I560I, USA, Tel 7249722703, Email leah1985@comcast.net

Received: February 26, 2015 | Published: June 03, 2015

\section{Opinion}

One of the greatest challenges for our public today is coping with our weight. Our nation continues to grow at a rapid rate every year with our increasingly busy life-styles, fast food restaurants, and sedentary behaviors. And what is worse, we are passing this on to our children as being natural. But what exactly does obesity mean and is it an immediate death sentence? Obesity is a clinical diagnosis for someone who has a body mass index (BMI) of greater than 30, which is computed using your height and weight. This BMI indicator does not measure fat versus muscle mass, but is a guideline for Registered Dietitian Nutritionists (RDN) and Registered Dietetic Technicians (DTR) to begin nutritional interventions. Once an individual hits the BMI status of 30 or more, over a period of time, this can cause other health problems to arise. Type 2 Diabetes and heart problems can occur from obesity in conjunction with a sedentary life style. But what about those people who eat healthy, exercise, and are obese? These individuals may not be at a high risk but need to seek advice from their physician because there may be another underlying problem.
So what can we do about this epidemic? Bottom line, eat healthy. When I discuss eating healthy with people, they get nervous about that word 'healthy'. It is simple. Follow the Myplate pyramid and eat a variety of foods. Fresh fruits, vegetables, lean meats, dairy, wholewheat grains and mono or polyunsaturated fats. If it is processed, consume it in moderation. If you are unsure if it is processed, look at the food label. The food label needs to list all natural ingredients on it. Anything that you cannot pronounce is probably not good for you.

The continued growth of obesity in the public can be dealt with. By turning to a RDN or DTR for nutrition advice is the best step in the right direction to help put an end to this epidemic.

\section{Acknowledgements}

None.

\section{Conflict of interest}

The author declares no conflict of interest. 